\title{
Fiabilidad y validez de la batería de evaluación del deterioro grave, versión abreviada (SIB-s), en pacientes con demencia en España
}

Isabel Cruz-Orduña, Luis F. Agüera-Ortiz, Ignacio Montorio-Cerrato, Beatriz León-Salas, M. Cristina Valle de Juan, Pablo Martínez-Martín

Introducción. Las personas con demencia progresiva evolucionan hacia un estado donde los tests neuropsicológicos tradicionales dejan de ser eficaces. La batería de evaluación del deterioro grave, en su forma completa (SIB) y abreviada (SIB-s), se desarrolló para evaluar el estado cognitivo de pacientes con demencia avanzada.

Objetivo. Evaluar los atributos psicométricos de la SIB-s en población española.

Pacientes y métodos. Estudio transversal de 127 pacientes con demencia (86,6\%, mujeres; edad media: 82,6 $\pm 7,5$ años) evaluados con la SIB-s y las siguientes medidas: escala de deterioro global, miniexamen cognitivo (MEC), miniexamen del estado mental grave (SMMSE), índice de Barthel y escala del estado funcional.

Resultados. La puntuación media total de la SIB-s fue de 19,1 $\pm 15,34$ (rango: 0-48). Efectos suelo y techo < 20\%. El análisis factorial identificó un único factor que explica el $68 \%$ de la varianza total de la escala. La consistencia interna fue alta ( $\alpha$ de Cronbach: 0,96). La correlación ítem-total corregida osciló entre 0,27 y 0,83, y la homogeneidad de los ítems fue de 0,43. La fiabilidad test-retest e interevaluador fue satisfactoria (coeficiente de correlación intraclase: 0,96 y 0,95, respectivamente), así como la validez de constructo convergente con otras medidas cognitivas (MEC: 0,83; sMMSE: 0,9). La SIB-s mostró una correlación moderada con escalas cognitivas de dependencia funcional (índice de Barthel: 0,48; FAST: $-0,74)$. El error estándar de la medida fue de 3,07 para el total de la escala.

Conclusiones. La SIB-s es un instrumento fiable y válido, relativamente breve, para evaluar a pacientes con demencia avanzada en la población española.

Palabras clave. Batería de evaluación del deterioro cognitivo grave versión abreviada (SIB-s). Demencia avanzada. Deterioro cognitivo grave. Enfermedad de Alzheimer. Fiabilidad. Test neuropsicológico. Validez.

\section{Introducción}

La mayoría de los estudios sobre demencia se ha centrado en los estadios leves y moderados. Sin embargo, son más limitados los trabajos que consideran las fases avanzadas de la enfermedad, pese a que todas las personas con demencia, especialmente de tipo Alzheimer, que viven lo suficiente progresan hacia un estado de un intenso declive funcional y cognitivo. La elevada comorbilidad, una mayor dependencia funcional y problemas conductuales característicos son los síntomas que provocan un mayor gasto en medidas de atención a la dependencia $[1,2]$. A medida que el deterioro cognitivo progresa, disminuye la importancia del diagnóstico sindrómico y aumenta la importancia del seguimiento evolutivo, la discriminación entre niveles de gravedad, la medición de los efectos terapéuticos y, como consecuencia, cambia la utilidad de los instrumentos de evaluación. Por ello, la evaluación de un trastorno como la demencia debe ir adaptándose a cada uno de los estadios en que la enfermedad va progresando, dado que los instrumentos que son aplicados a los pacientes que se encuentran en los estadios iniciales de la demencia pierden validez y dejan de ser útiles en las fases más avanzadas $[1,3,4]$.

La mayoría de los instrumentos para la evaluación de la demencia ha sido diseñada para ser aplicada en fases leves o moderadas. Los avances médicos y en investigación y el desarrollo de los cuidados están aumentando el número de pacientes con demencia que llegan a un estado avanzado de la enfermedad. Además, hay evidencia de que en estas fases avanzadas de la demencia existe capacidad cognitiva, que puede detectarse con tests específicos que sean más sensibles al estado cognitivo y que, al mismo tiempo, sean capaces de dar cuenta de la heterogeneidad de la ejecución cognitiva en las fases avanzadas de la enfermedad. La realidad clínica muestra que se cuenta con insuficientes instrumen-
Unidad de Investigación del Proyecto Alzheimer; Fundación CIEN-Fundación Reina Sofía; Instituto de Salud Carlos III; Centro Alzheimer Fundación Reina Sofía (I. Cruz-Orduña, L.F. Agüera-Ortiz, B. León-Salas, M.C. Valle de Juan). Centro de Investigación Biomédica en Red de Salud Mental, CIBERSAM (L.F. Agüera-Ortiz). Facultad de Psicología; Universidad Autónoma de Madrid (I. Montorio-Cerrato). Centro Nacional de Epidemiología y CIBERNED; Instituto de Salud Carlos III (P. Martínez-Martín). Madrid, España.

Correspondencia:

Dra. Isabel Cruz Orduña. Unidad de Investigación Proyecto Alzheimer. Centro Alzheimer Fundación Reina Sofía. Fundación CIEN Valderrebollo, 5. E-28031 Madrid.

Fax: +34 913852118.

E-mail:

isabelpsicol@hotmail.com

Financiación:

Ninguna: trabajo realizado como parte de la actividad reglada del Centro Alzheimer Fundación Reina Sofía.

Aceptado tras revisión externa: 30.09.14.

Cómo citar este artículo: Cruz-Orduña I, Agüera-Ortiz LF, Montorio-Cerrato I, León-Salas B, Valle de Juan MC, Martínez-Martín P. Fiabilidad y validez de la batería de evaluación del deterioro grave, versión abreviada (SIB-s), en pacientes con demencia en España. Rev Neurol 2015; 60: 1-9.

(c) 2015 Revista de Neurología 
tos válidos y fiables para evaluar el estado mental en estos pacientes.

Debido a todo lo expuesto, se hacen necesarios instrumentos especialmente diseñados para aumentar la capacidad de discriminación entre distintos niveles de deterioro, especialmente detectar rendimientos cognitivos y funcionales muy bajos y así evitar el característico efecto suelo $[1,5,6]$. Con este fin se han desarrollado baterías cognitivas específicas para los estadios más graves de la demencia, entre las que destacan el test de deterioro grave (Test for Severe Impairment) [7], el perfil de deterioro cognitivo grave (Severe Cognitive Impairment Profile) [8], la batería de evaluación del deterioro grave ( $\mathrm{Se}$ vere Impairment Battery, SIB) [9] y el miniexamen del estado mental grave (Severe Mini Mental Status Examination, sMMSE) [10]. En España contamos con cuatro herramientas con validación a la lengua castellana [11-14].

La SIB es una prueba cognitiva desarrollada específicamente para valorar a pacientes con tal grado de deterioro cognitivo que no puedan completar los tests neuropsicológicos habituales [9]. La batería está formada por nueve dominios, cada uno de los cuales cuenta con una puntuación independiente. Este test ha sido validado en distintos países e idiomas, incluyendo una validación al inglés [9], castellano [13], francés [5], italiano [15], japonés [16,17] y noruego [18]. Una de las características más interesantes de la SIB es su utilidad en la medición de cambios longitudinales en la función cognitiva en un rango de pacientes que oscila desde moderado a grave $[4,19]$. Como limitaciones, se han señalado un posible efecto techo en fases de demencia moderada [1], un tiempo largo de aplicación, así como la necesidad de personal entrenado y de material específico para su aplicación [11]. Posteriormente, Saxton et al desarrollaron una versión abreviada de la SIB (SIB-s), que mantiene la validez de contenido de la escala original [20]. Existe validación de esta escala abreviada al francés [21], inglés [20], japonés [22] y neerlandés [23].

Este trabajo tiene como objetivo examinar las características psicométricas de la versión en español de la SIB-s en pacientes institucionalizados con demencia avanzada.

\section{Pacientes y métodos}

\section{Diseño}

Estudio observacional, unicéntrico, transversal, con retest.

\section{Muestra}

Los pacientes proceden de la residencia $(85,8 \%)$ y del centro de día $(14,2 \%)$ para sujetos con demencia degenerativa del Centro Alzheimer Fundación Reina Sofía (CAFRS). Todos ellos fueron evaluados siguiendo el protocolo semestral del propio CAFRS [24]. La muestra del estudio estaba formada por 127 pacientes (110 mujeres y 17 hombres) con un diagnóstico de demencia basado en los criterios del $\mathrm{Ma}$ nual diagnóstico y estadístico de los trastornos mentales, cuarta edición, texto revisado [25]. El criterio de inclusión fue que en el momento de la evaluación hubieran recibido un diagnóstico de demencia y una puntuación $\leq 12$ en el miniexamen cognitivo-30 (MEC-30) [1,26]. Los criterios de exclusión del estudio fueron enfermedad grave concomitante, encamamiento, imposibilidad de comunicación, ausencia de contacto o estado confusional.

\section{Protocolo de evaluación}

\section{Deterioro global}

- Escala de Deterioro Global (Global Deterioration Scale, GDS). Instrumento de estadificación de las diferentes fases evolutivas de la demencia. Valora las alteraciones funcionales y cognitivas del paciente [27]. Consta de siete estadios de gravedad de la demencia, que oscilan desde normal (GDS 1) a demencia grave (GDS 7).

\section{Cognición}

- MEC. Versión en castellano [28] del Mini Mental Status Examination [29]. Instrumento diseñado para la evaluación del deterioro cognitivo en la población general. El MEC cumple criterios de fiabilidad, validez de contenido, de procedimiento y de construcción.

- Miniexamen del estado mental grave. Validación española [11] de la versión estadounidense del sMMSE [10]. El sMMSE es un instrumento de evaluación del deterioro cognitivo avanzado que amplía el rango inferior de medida del MEC, evitando el 'efecto suelo'. Los dominios cognitivos evaluados son: información sobreaprendida, funciones visuoespaciales simples, funciones ejecutivas, funciones lingüísticas simples y fluencia semántica simple. El rango de puntuación es de 0-30 puntos. Es un test considerado válido y fiable, así como rápido y fácil de administrar.

- SIB-s [20]. La selección de los ítems de la SIB-s está basada factorialmente sobre los ítems originales de la versión completa. La SIB-s se administra en unos 15 minutos, la mitad de tiempo 
que la escala completa. Consta de 26 preguntas agrupadas en los mismos nueve dominios que la escala original. El rango posible de puntuación oscila de 0 a 50 , con un máximo de dos puntos para 24 de los ítems y de un punto para dos ítems (19 y 38). Mantiene las propiedades psicométricas de validez y fiabilidad de la escala original. Los autores de esta versión reducida concluyen que es una prueba adecuada para sujetos con demencia avanzada. En el presente trabajo, la SIB-s de Saxton et al [20] se administró sin ninguna modificación.

\section{Estado funcional}

- Índice de Barthel [30]. Se diseñó para medir la evolución de sujetos con procesos neuromusculares y musculoesqueléticos en un hospital para enfermos crónicos de Maryland. Valora la capacidad de una persona para realizar de forma dependiente $\mathrm{o}$ independiente 10 actividades de la vida diaria básicas. Se asigna una puntuación $(0$, 5,10 o 15) en función del tiempo empleado en su realización y la necesidad de ayuda para llevarla a cabo. La puntuación final varía de 0 (máxima dependencia) a 100 (máxima independencia).

- Escala del estado funcional (Functional Assessment Staging, FAST) [31]. Escala de evaluación que se aplica, en general, como una extensión de la escala GDS. Presenta una descripción de siete fases características de la evolución, desde la normalidad hasta las fases más graves de la demencia tipo Alzheimer. El objetivo de la escala es evaluar las pérdidas funcionales en el envejecimiento normal y la demencia tipo Alzheimer. La FAST es especialmente interesante en la evaluación de las fases más avanzadas de este tipo de demencia. La fase GDS 6 se corresponde con cinco subfases en la FAST, y la GDS 7 se corresponde con seis.

\section{Procedimiento}

Todos los pacientes que formaban parte del protocolo semestral del CAFRS y cumplían con los criterios de inclusión fueron valorados, además, con la escala SIB-s. Los sujetos fueron evaluados con el MEC y la SIB-s por dos psicólogas entrenadas en la administración y puntuación de ambas baterías y ciegas a los resultados del resto de escalas que fueron administradas por dos neurólogos en el mismo mes. La segunda aplicación de la SIB-s se realizó en una media de tiempo de 19,98 \pm 13,89 días (rango: 7-54 días) tras la primera aplicación, con objeto de contrastar la estabilidad de la escala.

\section{Aspectos éticos}

Todos los sujetos o sus representantes legales habían firmado un consentimiento informado para el estudio, previamente aprobado por el Comité Ético de la Fundación del Centro de Investigación de Enfermedades Neurológicas (Fundación CIEN).

\section{Análisis estadístico}

Se utilizaron estadísticos descriptivos para las variables sociodemográficas, históricas y clínicas, y se determinaron los siguientes atributos psicométricos de la escala SIB-s, según estándares habituales [32].

\section{Viabilidad y aceptabilidad}

Para examinar la viabilidad y aceptabilidad se analizó el porcentaje de datos perdidos, el rango de puntuaciones mínimas y máximas, las medidas de tendencia central, el efecto techo y suelo, y, por último, la asimetría. La calidad de los datos se consideró satisfactoria si más del 90\% de los datos de la SIB-s eran computables $[33,34]$. Se siguió el criterio de imputación cuando su pérdida se dio en menos del $25 \%$ de los datos [35]. Se determinaron las diferencias entre media y mediana (límite arbitrario, el 10\% del máximo posible de la puntuación) [36], el efecto suelo y techo (un 15\% de máximo aceptado en ambos casos) [37] y la asimetría (skewness, límites, -1 a +1) [39].

\section{Fiabilidad}

La consistencia interna fue evaluada usando el coeficiente de Cronbach (criterio estándar $\geq 0,7$ ) [39], la correlación ítem-total corregida (criterio estándar $\geq 0,4)[40]$ y el índice de homogeneidad de los ítems (criterio estándar $\geq 0,3$ ) [41]. La fiabilidad test-retest $(n=50)$ e interevaluador $(n=50)$ de los ítems se determinaron por el índice kappa (con pesos lineales para puntuaciones 0-2) (criterio > $0,6)$. La fiabilidad de las puntuaciones totales de los dominios y la escala se determinaron por el coeficiente de correlación intraclase (ICC) (criterio $>0,7)[32]$.

\section{Validez}

Se obtuvo la estructura factorial de la escala mediante un análisis factorial de componentes principales, sobre las puntuaciones de los nueve dominios que componen la escala. La validez de criterio se analizó mediante la correlación de la SIB-s con la SIB. Se utilizó como criterio de calidad psicométrica una correlación entre la escala SIB-s y dos escalas cognitivas (MEC y sMMSE) $\geq 0,5$ [42]. Asimismo, se estableció la validez convergente de la SIB-s 
Tabla I. Características de la muestra $(n=127)$.

\begin{tabular}{|c|c|c|c|c|c|}
\hline \multirow{2}{*}{ Sexo } & Mujer & $110(86,6 \%)$ & \multirow{5}{*}{$\begin{array}{l}\text { Diagnóstico } \\
\text { etiológico } \\
\text { (cont.) }\end{array}$} & Demencia por cuerpos de Lewy & $8(6,2 \%)$ \\
\hline & Hombre & $17(13,4 \%)$ & & Demencia frontotemporal & $2(1,6 \%)$ \\
\hline \multirow{4}{*}{ Edad } & $\leq 74$ años & $16(12,6 \%)$ & & Enfermedad de Parkinson & $3(2,4 \%)$ \\
\hline & 75-84 años & $58(45,7 \%)$ & & & \\
\hline & & & & Incierto & $2(1,6 \%)$ \\
\hline & $\geq 85$ años & $53(41,7 \%)$ & \multirow{4}{*}{ GDS } & 5. moderado & $9(71 \%)$ \\
\hline \multirow{7}{*}{ Educación } & Iletrados & $15(11,8 \%)$ & & 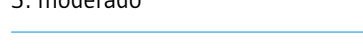 & $y(1,1 \% 0)$ \\
\hline & Lee, pero no escribe & $1(0,8 \%)$ & & 6: moderadamente grave & $55(43,3 \%)$ \\
\hline & Lee y escribe recados/notas & $17(13,4 \%)$ & & 7: grave & $63(49,6 \%)$ \\
\hline & Educación primaria & $86(67,7 \%)$ & \multirow{10}{*}{ FAST } & 5: demencia moderada & $4(3,2 \%)$ \\
\hline & Bachiller elemental & $6(4,7 \%)$ & & 6a: ayuda a vestirse & $3(2,4 \%)$ \\
\hline & Bachiller superior & $2(1,6 \%)$ & & 6b: ayuda a bañarse & $11(8,7 \%)$ \\
\hline & Universitarios & 0 & & 6c: ayuda a ir al baño & $18(14,3 \%)$ \\
\hline \multirow{4}{*}{ Estado civil } & Soltero & $8(6,3 \%)$ & & $6 \mathrm{~d}$ : incontinencia urinaria & $4(3,2 \%)$ \\
\hline & Casado & $40(31,5 \%)$ & & 6e: incontinencia fecal & $32(25,4 \%)$ \\
\hline & Viudo & $76(59,8 \%)$ & & 7a: lenguaje con escasas palabras & $32(25,4 \%)$ \\
\hline & Divorciado/separado & $3(2,3 \%)$ & & 7b: lenguaje con una palabra & $8(6,3 \%)$ \\
\hline \multirow{3}{*}{$\begin{array}{l}\text { Diagnóstico } \\
\text { etiológico }\end{array}$} & Probable enfermedad de Alzheimer & $78(61,4 \%)$ & & 7c: no deambula & $12(9,5 \%)$ \\
\hline & Posible enfermedad de Alzheimer & $16(12,6 \%)$ & & 7e: no sonríe & $2(1,6 \%)$ \\
\hline & $\begin{array}{l}\text { Enfermedad de Alzheimer } \\
\text { con enfermedad cerebrovascular }\end{array}$ & $18(14,2 \%)$ & FAST: escala & astado funcional; GDS: escala de deteri & global. \\
\hline
\end{tabular}

mediante la correlación de sus puntuaciones con las obtenidas en las dos dimensiones funcionales evaluadas (Barthel y FAST) con el mismo criterio $r_{\mathrm{s}} \geq 0,5$ [42]. También se determinó la validez interna (correlación interdominios; estándar: 0,3-0,7) [43]. Se utilizó el coeficiente de correlación de Spearman por no cumplirse las asunciones para uso de pruebas paramétricas. El análisis para la detección de diferencias estadísticamente significativas entre los tres grupos de la GDS $(5,6$ y 7$)$ en la SIB-s se realizó mediante la prueba de Kruskal-Wallis.

\section{Precisión}

La precisión se estimó por medio del error estándar de la medida (SEM) [37] para la puntuación total de la SIB-s $\left(\mathrm{SEM}=\mathrm{DE}_{\text {basal }} \times \sqrt{1-\mathrm{ICC}_{\text {test-retest }}}\right)$. Se consideró como límite mínimo aceptable un valor inferior a 0,5 DE basal [36].

\section{Resultados}

\section{Características de la muestra}

La muestra está formada por 127 pacientes con demencia, siendo el diagnóstico de enfermedad de Alzheimer posible o probable (74\%) el más frecuente. Respecto al grado de deterioro, en el 92,9\%, los pacientes presentan un deterioro moderadamente grave o grave. Para consultar las características de la muestra y los descriptivos de las escalas administradas puede acudirse a las tablas I y II. Entre los pacientes que pertenecían al centro de día y los institucionalizados no existieron diferencias significativas en escolaridad, sexo, GDS, años de evolución de la enfermedad y puntación total del MEC, si bien sí existieron diferencias significativas en edad, siendo más jóvenes los sujetos del centro de día. 
Tabla II. Puntuaciones de las escalas administradas.

\begin{tabular}{lcccc}
\hline & Media & $\begin{array}{c}\text { Desviación } \\
\text { estándar }\end{array}$ & $\begin{array}{c}\text { Rango } \\
\text { observado }\end{array}$ & $\begin{array}{c}\text { Rango } \\
\text { teórico }\end{array}$ \\
\hline SIB & 37,15 & 30,95 & $0-96$ & $0-100$ \\
\hline MEC & 4,09 & 3,92 & $0-12$ & $0-30$ \\
\hline SMMSE & 10,68 & 8,08 & $0-28$ & $0-30$ \\
\hline Barthel & 35,33 & 28,26 & $0-98$ & $0-100$ \\
\hline
\end{tabular}

MEC: miniexamen cognitivo; SIB: batería para el deterioro grave; SMMSE: miniexamen del estado mental grave.

\section{Características psicométricas}

\section{Viabilidad y aceptabilidad}

La tabla III muestra los datos de viabilidad y aceptabilidad de la escala y sus dimensiones tras la imputación de datos en cuatro casos (3,15\%; datos totalmente computables: 96,85\%). La puntuación media total de la SIB-s fue de 19,1 \pm 15,34 (mediana: 19; rango: 0-48), mostrando un efecto suelo marginal del $18,1 \%$ y un efecto techo menor del $15 \%$. En $36 / 127(28,3 \%)$ sujetos se obtuvo una puntuación de 0 en el MEC, y en el caso del sMMSE fueron 29/124 sujetos los que obtuvieron una puntuación de $0(23,4 \%)$. El percentil 50 o mediana de la puntuación total del MEC fue de 3. La media de la puntuación total de la SIB-s para pacientes con un $\mathrm{MEC} \leq 3(n=67)$ fue de 7,90 \pm 8,72 (rango: 0-29). Para los sujetos con una puntuación en el MEC > 3, la media en el SIB-s fue de 31,6 $\pm 10,8$ (rango: 8-48). La media y la varianza de la puntuación total de la SIB-s mostraron diferencias entre los dos grupos del MEC para cada muestra $(t=-13,71$; g.l. = 125; $p=0$; Levene $F=3,21 ; \mathrm{p}=0,08)$. No se encontró asimetría en ninguna dimensión de la SIB-s, a excepción de la dimensión 'construcción'. El tiempo medio de administración de la SIB-s fue de 9,6 minutos (rango: 5,5-15 minutos).

\section{Fiabilidad}

La tabla III muestra los datos de consistencia interna de las nueve dimensiones de la SIB-s y la escala total, y los datos de la estabilidad se pueden consultar en la tabla IV. El coeficiente $\alpha$ de Cronbach fue de 0,96. Los coeficientes de correlación ítem-total corregido fueron de 0,27 (ítem 'nombre del examinador_diferido') a 0,83 (ítem 'identificar color'). El índice de homogeneidad de los ítems fue de 0,43. La fiabilidad test-retest $(n=50)$ se examinó con el
Figura. Puntuación media en la batería de evaluación del deterioro grave, versión abreviada (SIB-s), según la intensidad de la demencia (escala de deterioro global, GDS).

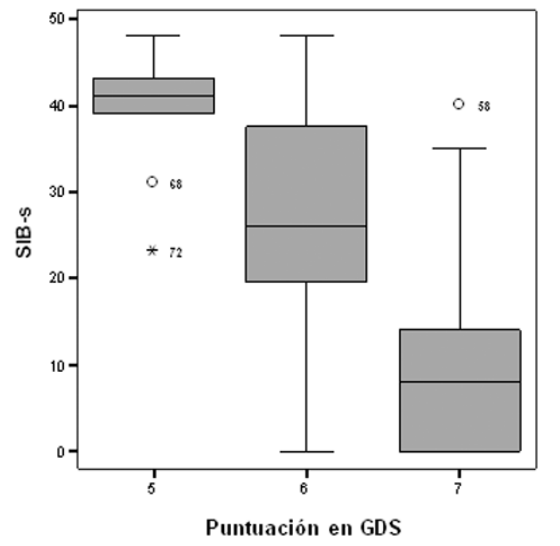

ICC, que resultó 0,96 para el total de la SIB-s (intervalo de confianza al 95\% = 0,95-0,97). El índice kappa para los ítems alcanzó valores entre 0,36 ('nombre del examinador_diferido') a 0,93 ('interacción social sigue directivas'). Para los dominios, el rango del ICC osciló de 0,71 (dominio 'memoria') a 0,95 (dominio 'orientación al nombre'). Respecto a la fiabilidad test-interobservador $(n=50)$, los valores kappa estuvieron entre 0,57 (ítem 'verbales') a 1 (ítem 'orientación al nombre'). Para los dominios, el ICC fue de 0,81 (dominio 'praxias') a 1 (dominio 'orientación al nombre'), y para el total de la escala fue de 0,95.

\section{Validez}

Los resultados del análisis factorial muestran que emerge un único factor que explica el $68 \%$ de la varianza total de la escala, con un autovalor del 6,1\%. El valor del índice Kaiser-Meyer-Olkin de adecuación de la muestra fue de $0,91\left(\chi_{(36)}^{2}=999 ; p=0\right)$, mostrando una excelente adecuación muestral. Asimismo, la SIB-s mostró una elevada correlación con la escala SIB global $\left(r_{\mathrm{s}}=0,99\right)$ y, como se hipotetizó, una fuerte validez convergente con otras medidas desarrolladas para la evaluación del estado cognitivo (MEC: $r_{\mathrm{s}}=0,83$; sMMSE: $r_{\mathrm{s}}=0,9$ ). La correlación entre los dominios de la SIB-s oscila desde 0,4 hasta 0,84. La puntuación total de la SIB-s mostró una correlación moderada con la puntuación en Barthel $\left(r_{\mathrm{s}}=0,48\right)$ y una correlación elevada con la FAST $\left(r_{\mathrm{s}}=-0,74\right)$. Se observaron diferencias estadísticamente significativas en la SIB-s según el estadio de la demencia, con puntuaciones significati- 
Tabla III. Propiedades psicométricas de la SIB-s.

\begin{tabular}{|c|c|c|c|c|c|c|c|c|c|c|}
\hline & Media & Mediana & $\mathrm{DE}$ & Asimetría & Mínimo & Máximo & $\begin{array}{c}\text { Datos } \\
\text { perdidos (\%) }\end{array}$ & $\begin{array}{c}\text { Efecto } \\
\text { suelo (\%) }\end{array}$ & $\begin{array}{c}\text { Efecto } \\
\text { techo (\%) }\end{array}$ & $\begin{array}{c}\alpha \text { de Cronbach } \\
\text { (correlación ítem-total) }^{\text {a }}\end{array}$ \\
\hline Dominio 1. Interacción social & 2,56 & 4 & 1,74 & $-0,584$ & 0 & 4 & 0 & 26,0 & 53,5 & $0,76(0,75-0,77)$ \\
\hline Dominio 2. Memoria & 1,94 & 2 & 1,95 & 0,511 & 0 & 7 & 0 & 41,7 & 0,8 & $0,51(0,27-0,69)$ \\
\hline Dominio 3. Orientación & 1,94 & 2 & 1,46 & $-0,139$ & 0 & 4 & 0 & 27,0 & 15,6 & $0,57(0,71-0,82)$ \\
\hline Dominio 5. Atención & 1,08 & 1 & 0,86 & $-0,153$ & 0 & 2 & 0 & 33,1 & 40,9 & 0,82 \\
\hline Dominio 6. Praxis & 1,61 & 0 & 1,89 & 0,400 & 0 & 4 & 0 & 55,1 & 36,2 & $0,60(0,60-0,61)$ \\
\hline Dominio 7. Habilidad visuoespacial & 1,54 & 0 & 1,71 & 0,411 & 0 & 4 & 0 & 51,2 & 24,4 & $0,76(0,70-0,83)$ \\
\hline Total SIB-s & 19,1 & 19 & 15,34 & 0,254 & 0 & 48 & 4,0 & 18,1 & 1,6 & 0,96 \\
\hline
\end{tabular}

DE: desviación estándar; SIB-s: batería de evaluación del deterioro grave, versión abreviada. ${ }^{a}$ Media (rango).

vamente más elevadas en los residentes en estadios menos graves y más bajas en los residentes en estadios graves de la demencia (Kruskal-Wallis, $p=0$ ) (Figura).

\section{Precisión}

El SEM de la SIB-s fue de 3,07 (0,5 DE basal = 7,67).

\section{Discusión}

El objetivo del estudio fue llevar a cabo la validación de la versión española de la SIB-s en pacientes con demencia avanzada.

Respecto a las capacidades psicométricas de la escala, la aceptabilidad fue satisfactoria para todas sus dimensiones y no existe asimetría destacable en su distribución, excepto para la dimensión 'construcción' (Tabla III). Un aspecto destacable de la SIB-s es que la escala supera una de las principales limitaciones de este tipo de instrumentos, al presentar una ausencia de efecto techo y un mínimo efecto suelo, lo que tiene diversas ventajas tanto para la investigación como para la práctica clínica, y hace posible que la escala posea una satisfactoria sensibilidad al cambio. Esta propiedad es necesaria para las medidas evaluativas, ya que la acumulación de puntuaciones en los extremos sin capacidad para diferenciar entre estados diferentes impide determinar modificaciones en el constructo evaluado [44].

La escala presenta una consistencia interna satisfactoria (Tabla III). El $\alpha$ de Cronbach es óptima $(0,96)$ y resulta similar a la de otros estudios de validación realizados con la SIB-s [20-23]. Sin embargo, valores muy elevados de $\alpha(>0,95)$ pueden indicar redundancia y se obtienen habitualmente en escalas con un gran número de ítems [32]. La correlación ítem-total corregida y el índice de homogeneidad de los ítems presentan, en conjunto, valores superiores a los umbrales teóricos exigidos, reflejando coherencia entre los ítems dentro de cada dimensión. En nuestra escala, todos los ítems correlacionan con el total por encima de 40 , excepto el ítem 14 ('recordar nombre del examinador de forma diferida'; $r=0,27)$. Esto coincide con los resultados obtenidos en otros trabajos de validación [21-23]. Hugonot-Diener et al [21] argumentan, al respecto, que este ítem 'atípico' fue incluido en la escala por motivos clínicos.

Respecto a la fiabilidad test-retest de los dominios, las puntuaciones oscilan entre 0,71 y 0,95 ( $\mathrm{Ta}$ bla IV). Todos ellos pueden considerarse satisfactorios como para afirmar que cada uno de los dominios tiene una suficiente estabilidad temporal. $\mathrm{Si}$ comparamos los resultados obtenidos por la escala SIB-s con la fiabilidad test-retest obtenida en otro 
estudio de validación, observamos que las puntuaciones son similares [22]. Estos datos sugieren, finalmente, que este instrumento posee una excelente reproductibilidad.

Los coeficientes de fiabilidad entre evaluadores y para cada uno de los ítems de la SIB-s están por encima del umbral mínimo establecido (Tabla IV), excepto en el ítem 9b ('ahora déme la otra mano'). Este hecho es indicativo de claridad y aplicabilidad en los procedimientos de puntuación de la escala, y permite concluir que los resultados no dependen significativamente de la subjetividad de los diferentes evaluadores, sino que son invariantes respecto a los evaluadores. Hasta donde nosotros sabemos, no existen otros estudios de fiabilidad entre evaluadores con la SIB-s.

Los resultados del análisis factorial muestran que la SIB-s es una escala homogénea y unidimensional, donde todos sus dominios saturan en el mismo factor y, por tanto, es coherente, es decir, parece medir la misma dimensión de base: la cognición. Como era esperable, existe una fuerte asociación entre esta escala y la SIB, como se ha observado en estudios previos [20-22]. En la versión original de Saxton et al [20], la correlación de Pearson entre la puntuación total de la SIB-s y la versión original fue de $0,974$ ( $p<0,001)$. Otros trabajos de validación muestran correlaciones entre la SIB-s y el MEC cuyos valores oscilan desde 0,83 a 0,91 [21-23]. De acuerdo con las hipótesis planteadas a priori en referencia a la validez de constructo, en nuestro estudio, la SIB-s también presenta una buena validez convergente con otras medidas cognitivas similares, como el MEC y el sMMSE. Todo ello nos vendría a indicar la utilidad de la escala para evaluar el componente cognitivo en las fases más avanzadas de las demencias. Asimismo, esta escala se asocia moderadamente con la capacidad funcional, aunque, en otros estudios, la correlación es algo mayor [22,23]. Esto podría deberse a que en nuestro estudio la escala utilizada para evaluar las actividades de la vida diaria no es específica para demencias. Por otro lado, encontramos una elevada correlación entre medidas generales del estado funcional en pacientes con demencia avanzada (medidas con la FAST) y el estado cognitivo (medido con la SIB-s), lo que indica que la ejecución en estos índices generales de funcionamiento cognitivo predice las capacidades funcionales [45]. El déficit cognitivo evaluado con la SIB-s se asocia a mayor gravedad de la demencia (Figura). En conjunto, toda esta evidencia vendría a apoyar la validez de constructo de la SIB-s como instrumento específico para la evaluación de demencia avanzada.

\begin{tabular}{lcc} 
Tabla IV. Estabilidad de la escala. & & \\
\hline & Fiabilidad test-restest & Fiabilidad interobservadores \\
\hline Dominio 1. Interacción social & $0,94(0,87-0,98)$ & $0,95(0,86-1,00)$ \\
\hline Dominio 2. Memoria & $0,71(0,64-0,80)$ & $0,84(0,73-0,87)$ \\
\hline Dominio 3. Orientación & $0,78(0,64-0,82)$ & $0,82(0,77-0,86)$ \\
\hline Dominio 4. Lenguaje & $0,93(0,87-0,96)$ & $0,93(0,91-0,97)$ \\
\hline Dominio 5. Atención & $0,84(0,62-0,88)$ & $0,88(0,81-0,93)$ \\
\hline Dominio 6. Praxis & $0,87(0,74-0,94)$ & $0,81(0,72-0,91)$ \\
\hline Dominio 7. Habilidad visuoespacial & $0,86(0,78-0,90)$ & $0,88(0,78-0,91)$ \\
\hline Dominio 8. Construcción & $0,82(0,72-0,94)$ & $0,91(0,88-0,97)$ \\
\hline Dominio 9. Orientación al nombre & $0,95(0,93-0,96)$ & $1,00(1,00-1,00)$ \\
\hline SIB-s & $0,96(0,95-0,97)$ & $0,95(0,93-0,98)$ \\
\hline & & 0 \\
\hline
\end{tabular}

SIB-S: batería de evaluación del deterioro grave, versión abreviada.

Uno de los principales inconvenientes en la aplicación de instrumentos de medición del deterioro cognitivo en personas con demencia hace referencia a las dificultades que estas personas experimentan para mantener la atención sostenida durante su administración [20,23]. La SIB-s tiene la ventaja de valorar de manera integrada el estado cognitivo de personas con demencia avanzada en un período razonable, estimado en 10-15 minutos. De esta manera, la SIB-s se convierte en un test apropiado a la hora de valorar a pacientes con demencia grave [23]. Otra de sus ventajas estriba en que los elementos utilizados pueden encontrarse en cualquier medio residencial.

Este estudio presenta algunas limitaciones. Por un lado, nos encontramos con el pequeño tamaño muestral del grupo de personas que están en un estadio GDS 5, comparado con los estadios 6 y 7, lo cual, aunque pueda suponer un peor conocimiento del comportamiento de la escala en estos pacientes menos avanzados, es coherente con el objetivo de la medición de la demencia avanzada y refuerza nuestros hallazgos en esta población. Por otro, consideramos que las investigaciones futuras deberían ir encaminadas a conocer la capacidad de esta escala para medir los cambios longitudinales en las funciones cognitivas y valorar qué ítems son los más sensibles a estos cambios. Finalmente, la muy elevada fiabilidad de la SIB-s, junto con la clara homo- 
geneidad de su estructura factorial, apunta a la posibilidad de cierta redundancia en el instrumento; así, podría plantearse para futuros trabajos una reducción de los ítems aún mayor de la escala sin alterar su estructura y sus buenas propiedades psicométricas, ya que, atendiendo al tipo de población al que va dirigida la SIB-s, es deseable una mayor brevedad de administración.

Los resultados del presente estudio sugieren que la SIB-s es un test fiable y válido en la evaluación de pacientes con demencia moderada-grave de forma relativamente breve, y mantiene la fiabilidad y validez de la SIB original sin un efecto techo y suelo significativos.

\section{Bibliografía}

1. Boller F, Verny M, Hugonot-Diener L, Saxton J. Clinical features and assessment of severe dementia. A review. Eur J Neurol 2002; 9: 125-36.

2. Sloane PD, Zimmerman S, Suchindran C, Reed P, Wang L, Boustani M, et al. The public health impact of Alzheimer's disease, 2000-2050: potential implication of treatment advances. Annu Rev Public Health 2002; 23: 213-31.

3. Robert PH, Mulin E, Mallea P, David R. Apathy diagnosis, assessment, and treatment in Alzheimer's disease. CNS Neurosci Ther 2010; 16: 263-71.

4. Schmitt FA, Ashford W, Ernesto C, Saxton J, Schneider LS, Clark CM, et al. The severe impairment battery: concurrent validity and the assessment of longitudinal change in Alzheimer's disease. The Alzheimer's Disease Cooperative Study. Alzheimer Dis Assoc Disord 1997; 11 (Suppl 2): S51-6.

5. Panisset M, Roudier M, Saxton J, Boller F. Severe Impairment Battery. A neuropsychological test for severely demented patients. Arch Neurol 1994; 51: 41-5.

6. Saxton J, Swihart AA. Neuropsychological assessment of the severely impaired elderly patient. Clin Geriatr Med 1989; 5: 531-43.

7. Albert M, Cohen C. The Test for Severe Impairment: an instrument for the assessment of patients with severe cognitive dysfunction. J Am Geriatr Soc 1992; 40: 449-53.

8. Peavy GM, Salmon DP, Rice VA, Galasko D, Samuel W, Taylor KI, et al. Neuropsychological assessment of severely demented elderly: the severe cognitive impairment profile. Arch Neurol 1996; 53: 367-72.

9. Saxton J, McGonogle-Gibson K, Swihart A, Miler V, Boller F. Assessment of the severely impaired patient: description and validation of a new neuropsychological test battery. Psychol Assess 1990; 2: 298-303.

10. Harrell LE, Marson D, Chatterjee A, Parrish JA. The Severe Mini-Mental State Examination: a new neuropsychologic instrument for the bedside assessment of severely impaired patients with Alzheimer disease. Alzheimer Dis Assoc Disord 2000; 14: 168-75.

11. Buiza C, Navarro A, Díaz-Orueta U, González MF, Álaba J, Arriola E, et al. Short evaluation of cognitive state in advanced stages of dementia: preliminary results of the Spanish validation of the Severe Mini-Mental State Examination. Rev Esp Geriatr Gerontol 2011; 46: 131-8.

12. Cejudo J, Torrealba E, García P, Auer S, Castrillo S, PeñaCasanova J. Validez y fiabilidad de las escalas M-OSPD en el estudio del deterioro cognitivo en pacientes con enfermedad de Alzheimer avanzada. Psicogeriatría 2011; 3: 87-92.

13. Llinàs-Regla J, Lozano-Gallego M, López OL, GudayolPortabella M, López-Pousa S, Vilalta-Franch J, et al. Validation of the Spanish version of the Severe Impairment Battery. Neurologia 1995; 10: 14-8.
14. Buiza C, Etxeberría I, Yanguas J, Yanguas E, Galdona N, González M. Estudio de validación y fiabilidad de una prueba de deterioro cognitivo grave en una población española. Rev Neurol 2005; 20: 611.

15. Pippi M, Mecocci P, Saxton J, Bartorelli L, Pettenati C, Bonaiuto S, et al. Neuropsychological assessment of the severely impaired elderly patient: validation of the Italian short version of the Severe Impairment Battery (SIB). Gruppo di Studio sull'Invecchiamento Cerebrale della Societa Italiana di Gerontologia e Geriatria. Aging (Milano) 1999; 11: 221-6.

16. Suh GH, Kang CJ. Validation of the Severe Impairment Battery for patients with Alzheimer's disease in Korea. Int J Geriatr Psychiatry 2006; 21: 626-32.

17. Ahn IS, Kim JH, Ku HM, Saxton J, Kim DK. Reliability and validity of the severe impairment battery (SIB) in Korean dementia patients. J Korean Med Sci 2006; 21: 506-17.

18. Bergh S, Selbaek G, Engedal K. Reliability and validity of the Norwegian version of the Severe Impairment Battery (SIB). Int J Geriatr Psychiatry 2008; 23: 896-902.

19. Wild KV, Kaye JA. The rate of progression of Alzheimer's disease in the later stages: evidence from the Severe Impairment Battery. J Int Neuropsychol Soc 1998; 4: 512-6.

20. Saxton J, Kastango KB, Hugonot-Diener L, Boller F, Verny M, Sarles CE, et al. Development of a short form of the Severe Impairment Battery. Am J Geriatr Psychiatry 2005; 13: 999-1005.

21. Hugonot-Diener L, Verny M, Devouche E, Saxton J, Mecocci P, Boller F. Abridged version of the severe impairment battery (SIB). Psychol Neuropsychiatr Vieil 2003; 1: 273-83.

22. Ahn IS, Kim JH, Saxton J, Kim DK. Reliability and validity of a short form of the Severe Impairment Battery in Korean Alzheimer's disease patients. Int J Geriatr Psychiatry 2007; 22: 682-7.

23. De Jonghe JF, Wetzels RB, Mulders A, Zuidema SU, Koopmans RT. Validity of the Severe Impairment Battery Short Version. J Neurol Neurosurg Psychiatry 2009; 80: 954-9.

24. Olazarán J, Agüera-Ortiz L, Osorio RS, León-Salas B, Dobato JL, Cruz-Orduña I, et al. Promoting research in advanced dementia: early clinical results of the Alzheimer Center Reina Sofia Foundation. J Alzheimers Dis 2012; 28: 211-22.

25. American Psychiatric Association. DSM-IV-TR: breviario. Criterios diagnósticos. Barcelona: Masson; 2003.

26. Schmitt FA, Wichems CH. A systematic review of assessment and treatment of moderate to severe Alzheimer's disease. Prim Care Companion J Clin Psychiatry 2006; 8: 158-9.

27. Reisberg B, Ferris SH, de Leon MJ, Crook T. The Global Deterioration Scale for assessment of primary degenerative dementia. Am J Psychiatry 1982; 139: 1136-9.

28. Lobo A, Saz P, Marcos G, Día JL, De la Cámara C, Ventura T, et al. Revalidation and standardization of the cognition mini-exam (first Spanish version of the Mini-Mental Status Examination) in the general geriatric population. Med Clin (Barc) 1999; 112: 767-74.

29. Folstein MF, Folstein SE, McHugh PR. 'Mini-mental state'. A practical method for grading the cognitive state of patients for the clinician. J Psychiatr Res 1975; 12: 189-98.

30. Mahoney FI, Barthel DW. Functional evaluation: the Barthel Index. Md State Med J 1965; 14: 61-5.

31. Reisberg B. Functional Assessment Staging (FAST). Psychopharmacol Bull 1988; 24: 653-9.

32. Terwee CB, Bot SD, De Boer MR, Van der Windt DA, Knol DL, Dekker J, et al. Quality criteria were proposed for measurement properties of health status questionnaires. J Clin Epidemiol 2007; 60: 34-42.

33. Marinus J, Visser M, Van Hilten JJ, Lammers GJ, Stiggelbout AM. Assessment of sleep and sleepiness in Parkinson disease. Sleep 2003; 26: 1049-54.

34. Van der Linden FA, Kragt JJ, Klein M, Van der Ploeg HM, Polman $\mathrm{CH}$, Uitdehaag BM. Psychometric evaluation of the multiple sclerosis impact scale (MSIS-29) for proxy use. J Neurol Neurosurg Psychiatry 2005; 76: 1677-81.

35. Shrive FM, Stuart H, Quan H, Ghali WA. Dealing with missing data in a multi-question depression scale: a comparison of imputation methods. BMC Med Res Methodol 2006; 6: 57. 
36. Martínez-Martín P, Rodríguez-Blázquez C, Abe K, Bhattacharyya KB, Bloem BR, Carod-Artal FJ, et al. International study on the psychometric attributes of the non-motor symptoms scale in Parkinson disease. Neurology 2009; 73: 1584-91.

37. McHorney CA, Tarlov AR. Individual-patient monitoring in clinical practice: are available health status surveys adequate? Qual Life Res 1995; 4: 293-307.

38. Hays RD, Anderson R, Revicki D. Psychometric considerations in evaluating health-related quality of life measures. Qual Life Res 1993; 2: 441-9.

39. Scientific Advisory Committee of the Medical Outcomes Trust. Assessing health status and quality-of-life instruments: attributes and review criteria. Qual Life Res 2002; 11: 193-205.

40. Ware JE Jr, Gandek B. Methods for testing data quality, scaling assumptions, and reliability: the IQOLA Project approach. International Quality of Life Assessment. J Clin Epidemiol 1998; 51: 945-52.
41. Eisen M, Ware JE Jr, Donald CA, Brook RH. Measuring components of children's health status. Med Care 1979; 17: 902-21.

42. Juniper E, Guyatt G, Jaeschke R. How to develop and validate a new quality of life instrument. In Spilker B, ed. Quality of life and pharmacoeconomics in clinical trials. New York: Lippincott-Raven; 1995. p. 49-56.

43. Hobart J, Lamping D, Fitzpatrick R, Riazi A, Thompson A The Multiple Sclerosis Impact Scale (MSIS-29): a new patient-based outcome measure. Brain 2001; 124: 962-73.

44. Fitzpatrick R, Davey C, Buxton MJ, Jones DR. Evaluating patient-based outcome measures for use in clinical trials. Health Technol Assess 1998; 2: i-iv, 1-74.

45. Galasko D, Schmitt F, Thomas R, Jin S, Bennett D. Detailed assessment of activities of daily living in moderate to severe Alzheimer's disease. J Int Neuropsychol Soc 2005; 11: 446-53.

\section{Reliability and validity of the Severe Impairment Battery, short form (SIB-s), in patients with dementia} in Spain

Introduction. People with progressive dementia evolve into a state where traditional neuropsychological tests are not effective. Severe Impairment Battery (SIB) and short form (SIB-s) were developed for evaluating the cognitive status in patients with severe dementia.

Aim. To evaluate the psychometric attributes of the SIB-s in patients with severe dementia.

Patients and methods. 127 institutionalized patients (female: 86.6\%; mean age: $82.6 \pm 7.5$ years-old) with dementia were assessed with the SIB-S, the Global Deterioration Scale (GDS), Mini-Mental State Examination (MMSE), Severe MiniMental State Examination (sMMSE), Barthel Index and FAST.

Results. SIB-s acceptability, reliability, validity and precision were analyzed. The mean total score for scale was $19.1 \pm 15.34$ (range: 0-48). Floor effect was $18.1 \%$, only marginally higher than the desirable $15 \%$. Factor analysis identified a single factor explaining $68 \%$ of the total variance of the scale. Cronbach's $\alpha$ coefficient was 0.96 and the item-total corrected correlation ranged from 0.27 to 0.83 . The item homogeneity value was 0.43 . Test-retest and inter-rater reliability for the total score was satisfactory (ICC: 0.96 and 0.95 , respectively). The SIB-s showed moderate correlation with functional dependency scales (Barthel Index: 0.48, FAST: -0.74). Standard error of measurement was 3.07 for the total score.

Conclusions. The SIB-s is a reliable and valid instrument for evaluating patients with severe dementia in the Spanish population of relatively brief instruments.

Key words. Alzheimer's disease. Neuropsychological test. Reliability. Severe cognitive impairment. Severe dementia. Severe Impairment Battery-short version (SIB-s). Validity. 